\title{
Propriétés en hautes fréquences des transistors MOS à canal court. Analyse théorique des propriétés de la zone active
}

\author{
P. Rossel, G. Guégan et H. Martinot \\ Laboratoire d'Automatique et d'Analyse des Systèmes du Centre National de la Recherche Scientique, \\ 7, avenue du Colonel-Roche, 31400 Toulouse, France
}

(Reçu le 22 janvier 1979, révisé le 9 mai 1979, accepté le 15 mai 1979)

\begin{abstract}
Résumé. - On propose deux méthodes de détermination des paramètres hautes fréquences de la zone active du transistor MOS. Les effets de réduction de la mobilité des porteurs, particulièrement sensibles dans les transistors à canaux courts, sont pris en compte. La première méthode conduit à expliciter les facteurs de la matrice admittance sous forme de combinaisons de développements en séries infinies selon les puissances croissantes de la fréquence. La deuxième méthode permet d'obtenir des expressions analytiques dont le domaine de validité en fonction de la fréquence et des tensions de polarisation est précisé. Les facteurs $\left[y_{i j}\right]$ sont normalisés par introduction de variables réduites. Les effets de la réduction de mobilité et de saturation de la vitesse se traduisent par une diminution notable des parties réelles et imaginaires des facteurs admittances - notamment de l'admittance de transfert directe par rapport aux analyses classiques. Les résultats obtenus font apparaître la nécessité de tenir compte de cette réduction pour l'évaluation des caractéristiques hautes fréquences des transistors (D. MOS, V. MOS) de la nouvelle génération à canal court.
\end{abstract}

\begin{abstract}
In this paper, two methods are proposed for the determination of the high frequency parameters of the MOS transistor. The effects of the mobility reduction of the carriers as a result of the action of the longitudinal (short channel effect) and the transversal components of the electrical field are taken into account. The first method involves writing out the admittance matrix terms in the form of an infinite series expansion where the powers in the series increase with the frequency. The second method enables one to obtain analytical expressions with a precisely defined range of utility as regards frequency and polarisation voltages. The $y_{i j}$ terms are normalised by the introduction of reduced variables. The mobility reduction effects manifest themselves by an appreciable reduction in both the real and imaginary parts of the admittance terms (especially the direct transfer admittance) in comparison with the results deduced from the constant mobility analyses already proposed. The results obtained bring out the nead to take into account this mobility reduction in the evaluation of the high frequency characteristics of the new transistor MOS families (D. MOS and V. MOS).
\end{abstract}

1. Introduction position du problème. - 1.1 LES DIVERSES FAMILLES DE TRANSISTORS MOS POUR HAUTES FRÉQUENCES. - Dès 1966, le transistor à effet de champ à grille isolée est apparu comme pouvant avoir de bonnes caractéristiques pour l'amplification [1, 2, 3], dans la gamme des hautes fréquences. Depuis cette date de nombreux travaux ont été effectués pour mettre au point des processus d'élaboration permettant de réaliser des composants de plus en plus performants, utilisables dans une première étape dans la gamme VHF puis celle des UHF [4] et enfin plus récemment dans celle des hyperfréquences [5]. Les caractéristiques les plus intéressantes que présente le transistor MOS sont : un grand gain en puissance, une faible capacité de réaction entrée-sortie et une distorsion d'intermodulation extrêmement réduite, plus faible que celle que l'on obtient avec les tubes ou les transistors bipolaires. Par ailleurs, la mise au point de technologies dites à canal vertical a ouvert la voie au domaine de l'amplification de puissance, pour lequel le transistor MOS paraît être le concurrent sérieux du transistor bipolaire. L'évolution des processus s'est faite dans deux directions : (i) la première est celle de la définition de dispositifs à double grille, l'une jouant le rôle d'électrode de commande et l'autre le rôle d'écran ; la stabilité du gain peut ainsi être assurée même si celui-ci est supérieur à $30 \mathrm{~dB}$; (ii) la deuxième est celle de la réduction du canal par les processus d'implantation ionique $[6,7]$, de double diffusion verticale [5] ou horizontale [8]. Ainsi on peut distinguer deux grandes familles de dispositifs utilisables en hautes fréquences : 
— Les MOS à double grille sur silicium massif [9] ou sur silicium sur isolant $[10,11,12]$ particulièrement bien adaptés à l'amplification faible signal, à très grand gain, à bande étroite et bien résistants à la transmodulation. La gamme de fréquences d'utilisation va de quelques mégahertz (structures classiques) à $2 \mathrm{GHz}$ (structures implantées sur silicium sur isolant ou à double diffusion, D. MOS);

- Les MOS, à canal vertical (V. MOS) épitaxié [13] ou diffusé, réalisés par attaque isotropique [14] ou anisotropique [15] du silicium dont la particularité est de présenter une configuration $\mathrm{N}^{-} \mathrm{N}^{+}$de la diode de drain. Ces structures, plus adaptées à l'amplification large bande, sont utilisables en tant qu'éléments de puissance et leur limite maximale d'utilisation, dépendant de la configuration géométrique et technologique, peut dépasser le gigahertz.

\subsection{LES SCHÉMAS ÉQUIVALENTS ET LES PARAMÈTRES} HAUTES FRÉQUENCES. - Corrélativement aux travaux technologiques, plusieurs méthodes de calcul ont été décrites dans la littérature, durant ces dernières années, en vue de proposer les expressions mathématiques des paramètres hautes fréquences petits signaux des transistors MOS. Elles sont principalement basées sur des approximations de développements en série [16-20], des méthodes numériques de résolution des équations différentielles dynamiques $[23,24]$ ou sur la méthode matricielle de recherche des solutions de la ligne de transmission analogue au transistor [25]. Les principaux résultats ne sont applicables qu'aux transistors MOS à canal long, supérieur à $5 \mu \mathrm{m}$, élaborés sur un substrat de forte résistivité. Les effets particuliers du dopage de substrat sur la zone active ont été analysés par Das [26] d'une part et par Martinot et Rossel [27, 28] d'autre part. De plus l'action de la réduction de mobilité sous l'action du champ transversal au canal, très sensible dans les dispositifs à faible épaisseur d'oxyde a été pris en compte par ces derniers auteurs. Toutefois, l'effet du champ longitudinal - dans la direction source drain - sur la réduction de mobilité et la saturation de vitesse, d'autant plus important que le composant est prévu pour pouvoir monter en fréquence et que son canal est court, n'a jusqu'à ce jour jamais été considéré. Ceci est dû principalement à la complexité mathématique du problème. Ainsi, aussi bien dans le cas du D. MOS [5] que celui du V. MOS [14], la connaissance $\mathrm{du}$ fonctionnement de la structure se réduit à l'établissement d'un schéma équivalent synthétisé à partir de mesures expérimentales des paramètres $(S)$ ou $(Y)$ du transistor. Les éléments de ce schéma ne peuvent être corrélés aux paramètres technologiques et géométriques et par suite il est impossible de préciser les limitations fréquentielles et les performances limites potentielles de ces transistors.

1.3 BUTS DE L'ÉTUDE. - L'objectif de l'étude est double : d'une part une analyse théorique de l'effet complet de réduction de mobilité sous l'action du champ électrique sera effectuée dans ce premier article. D'autre part et dans un article futur, les résultats seront appliqués à la structure V. MOS; on analysera à ce propos ses limitations fondamentales et on montrera comment l'étude théorique conduit à une technique de simulation des amplificateurs, à partir des données de la technologie des transistors, particulièrement souple et puissante.

De façon plus précise nous proposons dans cette première partie une méthode de détermination des facteurs de la matrice admittance $\left(y_{i j}\right)$ en petits signaux et en hautes fréquences de la région active, c'est-à-dire du canal d'inversion du transistor MOS. Ce canal apparaît au droit de l'interface oxydesemiconducteur de substrat; ce substrat est de type opposé aux électrodes de source et de drain. L'effet des éléments dits parasites sera analysé dans la deuxième partie.

\section{Equations régissant le comportement dynamique} du canal. - La figure 1 représente une coupe idéalisée de la structure étudiée dans laquelle apparaissent les diverses électrodes que sont la grille, le substrat, la source et le drain. Ces deux dernières sont considérées comme constituant les limites, dans la direction horizontale, du canal d'inversion. En régime variable les tensions de polarisation de grille, source et drain sont notées $V_{\mathrm{G}}(t), V_{\mathrm{R}}(t), V_{\mathrm{D}}(t)$; le substrat constitue la référence des potentiels. Il en résulte en tout point du canal de coordonnée $y$, l'existence d'une différence de potentiel $\varphi(y, t)$ entre l'interface et le substrat, et dans
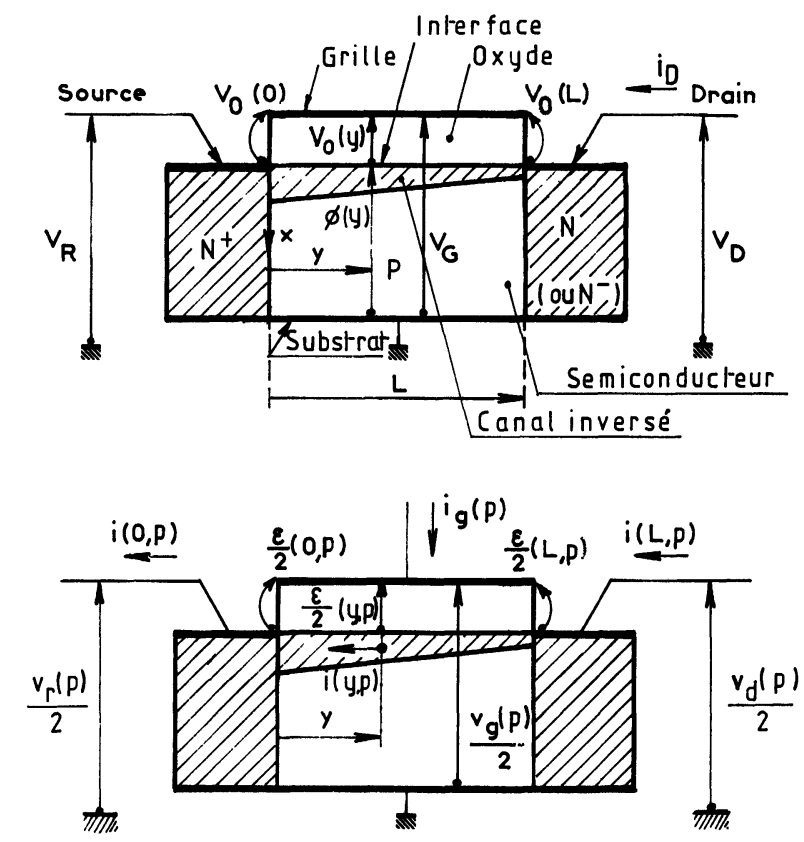

Fig. 1. - Coupe schématique du transistor, définition des variables en régime statique et en régime dynamique petits signaux.

[Structure of the MOS transistor defining the D.C. signals and the dynamic small signals.] 
une tranche de canal circule un courant $I(y, t)$. En introduisant les changements de variable suivants :

$$
\begin{aligned}
& V_{\mathrm{G}}^{\prime}(t)=V_{\mathrm{G}}(t)+\frac{Q_{\mathrm{SS}}}{C_{\mathrm{ox}}}-\varphi_{\mathrm{MS}} \\
& V(y, t)=V_{\mathrm{G}}^{\prime}(t)-\varphi_{\mathrm{S}}(y, t)
\end{aligned}
$$

où $t$ est le temps, $\varphi_{\mathrm{MS}}$ la différence des travaux de sortie entre la grille et le substrat, $Q_{\mathrm{ss}} / C_{\mathrm{ox}}$ le potentiel associé aux charges parasites d'oxyde et $C_{\text {ox }}$ la capacité d'oxyde de grille par unité de surface, et en prenant en compte la réduction de mobilité de porteurs dans la couche d'inversion par la relation [29] :

$$
\mu_{\mathrm{eff}}=\frac{\mu_{0}}{\left(1+\frac{\left|E_{x}\right|}{E_{\mathrm{C}}}\right) \cdot\left(1+\frac{\left|E_{y}\right|}{E_{0}}\right)}
$$

où $E_{x}(y, t)$ et $E_{y}(y, t)$ sont les composantes transversales et longitudinales du champ électrique et $E_{\mathrm{C}}$ et $E_{0}$ les champs critiques transversal et longitudinal on obtient l'équation différentielle du courant en régime variable :

$$
I(y, t)=-\mu_{0} Z C_{\mathrm{ox}} \frac{1}{\left[1-\frac{\partial V(y, t)}{\partial y} / E_{0}\right] \cdot\left[1+\frac{V(y, t)}{\psi}\right]} \cdot V(y, t) \cdot \frac{\partial V(y, t)}{\partial y}
$$

Dans cette relation les effets dus au dopage du substrat ont été négligés, $Z$ est la largeur de la grille et $\psi$ le potentiel de réduction de mobilité transversale [27] :

où $x_{0}$ est l'épaisseur de l'oxyde de grille.

$$
\psi=x_{0} \cdot E_{\mathrm{C}}
$$

Dans le cadre de l'hypothèse de petits signaux, par linéarisation on peut écrire :

$$
\begin{aligned}
& I(y, t)=I_{\mathrm{D}}+i(y, t) \\
& V(y, t)=V_{0}(y)+\frac{\varepsilon}{2}(y, t) .
\end{aligned}
$$

La relation (1) devient en négligeant les termes du $2^{\mathrm{e}}$ ordre :

$$
i(y, t)=I_{\mathrm{D}} \cdot\left[\frac{\psi+V_{0}(y)}{V_{0}(y)}\right] \cdot \frac{\partial}{\partial V_{0}(y)}\left[\left(\frac{V_{0}(y)}{\psi+V_{0}(y)}-\frac{I_{\mathrm{D}}}{\mu_{0} Z C_{\mathrm{ox}} \psi E_{0}}\right) \cdot \frac{\varepsilon}{2}(y, t)\right] .
$$

Par ailleurs, dans une tranche différentielle de canal de largeur dy, la perte de courant s'effectue à travers la capacité d'oxyde de grille suivant la loi :

$$
\frac{\partial i(y, t)}{\partial y}=-Z \cdot C_{\mathrm{ox}} \cdot \frac{\partial}{\partial t} \frac{\varepsilon}{2}(y, t)
$$

En se plaçant en régime alternatif sinusoïdal de pulsation $\dot{\omega}(p=j \omega)$, les expressions (2) et (3) fournissent, en introduisant les variables réduites suivantes :

$$
\omega_{0}=\frac{I_{\mathrm{D}}^{2}}{\mu_{0}\left(Z C_{\mathrm{ox}}\right)^{2} V_{0}(0)^{3}}
$$

et

$$
p^{\prime}=\frac{j \omega}{\omega_{0}}=\frac{p}{\omega_{0}}
$$

le système définissant le régime dynamique du transistor :

$$
\begin{gathered}
\frac{\partial^{2} i(y, p)}{\partial V_{0}(y)^{2}}-p^{\prime} \cdot\left[\frac{V_{0}(y) / V_{0}^{3}(0)}{1+V_{0}(y) / \psi}\right] \cdot i(y, p)=0 \\
\frac{\varepsilon}{2}(y, p)=\frac{1}{p^{\prime}} \cdot B\left(V_{0}(y)\right) \cdot \frac{\mathrm{d} i(y, p)}{\mathrm{d} V_{0}(y)}
\end{gathered}
$$

avec

$$
B\left(V_{0}(y)\right)=\frac{V_{0}^{3}(0)}{I_{\mathrm{D}} \psi}\left[\frac{V_{0}(y)}{\psi+V_{0}(y)}-\frac{I_{\mathrm{D}}}{\mu_{0} Z C_{\mathrm{ox}} \psi E_{0}}\right]^{-1}
$$

$V_{0}(0)$ et $V_{0}(L)$ sont égaux à $V_{0}(y)$ aux droits de la source $(y=0)$ et du drain $(y=L)$. 
3. Méthodes de résolution des équations différentielles régissant le comportement dynamique. - Le système non linéaire (4), (5) n'admet pas de solution explicitable simplement. Nous proposons deux méthodes de résolution qui consistent à rechercher le développement en série des solutions et les fonctions asymptotiques aux solutions en hautes fréquences.

3.1 RECHERCHE DES SOLUTIONS SOUS FORME DE DÉVELOPPEMENTS EN SÉRIE. - Cette méthode constitue une généralisation des calculs effectués par A. Van der Ziel et J. W. Ero [16] pour la détermination des paramètres fréquentiels des transistors à effet de champ à jonctions et des travaux effectués par E. N. Pronontarios [30, 31] relatifs au calcul des paramètres caractéristiques des simples lignes à constantes réparties non uniformes. Elle consiste à rechercher une solution a priori de la forme

$$
i(y, p)=a_{0}+a_{1} V_{0}(y)+\cdots+a_{n} V_{0}^{n}(y)+\cdots
$$

et à déterminer les coefficients $a_{n}$ par double dérivation de (6) puis report des quantités $i(y, p)$ et $\partial^{2} i(y, p) / \partial V_{0}(y)^{2}$ dans l'équation (4). Par identification on obtient la relation de récurrence :

$$
a_{n+3}=\frac{p^{\prime} a_{n} / V_{0}(0)^{3}-(n+1)(n+2) \cdot a_{n+2} / \psi}{(n+2)(n+3)}
$$

avec :

$$
a_{2}=0 \text {. }
$$

Cette série est convergente. Compte tenu de (7), on peut exprimer le coefficient $a_{n}$ en fonction des deux coefficients $a_{0}$ et $a_{1}$.

$$
\left.\begin{array}{rl}
a_{n}=a_{0}\left\{\frac{(-1)^{n-1} p^{\prime}}{V_{0}^{3}(0) \cdot n \cdot(n-1) \cdot \psi^{n-3}}+\left[\sum_{k=2}^{m} \frac{(-1)^{n-k} \cdot p^{\prime k}}{V_{0}^{3 k}(0) \cdot \psi^{n-3 k} \cdot n \cdot(n-1)} S_{0}(k, n)\right]\right\}+ \\
+a_{1}\left\{\frac{(-1)^{n} p^{\prime}}{V_{0}^{3}(0) \cdot n \cdot(n-1) \cdot \psi^{n-4}}+\left[\sum_{k=2}^{m^{\prime}} \frac{(-1)^{n-k+1} p^{\prime k}}{V_{0}(0)^{3 k} \cdot \psi^{(n-3 k-1)} \cdot n \cdot(n-1)} S_{1}(k, n)\right]\right\}
\end{array}\right\}
$$

avec :

$$
\begin{aligned}
m & =\text { partie entière de } n / 3 \\
m^{\prime} & =\text { partie entière de }(n-1) / 3
\end{aligned}
$$

les quantités $S_{0}$ et $S_{1}$ sont définies par l'expression :

$$
\begin{array}{r}
S_{i}(k, n)=\cdot\left[\sum_{i_{1}=n-3}^{i_{1}=3(k-1)+i} \frac{1}{i_{1} \cdot\left(i_{1}-1\right)} \times\left[\sum_{i_{2}=i_{1}-3}^{i_{2}=3(k-2)+i} \frac{1}{i_{2} \cdot\left(i_{2}-1\right)} \times\left[\sum_{i_{3}=i_{2}-3}^{i_{3}=3(k-3)+i} \frac{1}{i_{3} \cdot\left(i_{3}-1\right)} \times\right.\right.\right. \\
\times\left[\ldots \sum_{i_{j}=\left(i_{j-1}\right)-3}^{i_{j}=3[k-(j-1)-1]+i} \frac{1}{i_{j} \cdot\left(i_{j}-1\right)} \times\left[\cdots \times\left[\sum_{i_{k-1}=\left(i_{k-2}\right)-3}^{i_{k-1}=3+i} \frac{1}{i_{k-1} \cdot\left(i_{k-1}-1\right)}\right]\right]\right] \\
{[k \text { - crochets }]}
\end{array}
$$

Compte tenu de la formulation (9) du coefficient $a_{n}$, le courant $i(y, p)$ peut être écrit sous la forme :

$$
i(y, p)=a_{0} \cdot P\left(V_{0}(y), p^{\prime}\right)+a_{1} \cdot Q\left(V_{0}(y), p^{\prime}\right)
$$

avec :

$$
\begin{aligned}
& P\left(V_{0}(y), p^{\prime}\right)=1+\sum_{n=3}^{n=\infty}\left[\left(\frac{(-1)^{n-1} p^{\prime}}{V_{0}^{3}(0) \cdot \psi^{n-3} \cdot n \cdot(n-1)}+\sum_{k=2}^{m} \frac{(-1)^{n-k} p^{\prime k}}{V_{0}^{3 k}(0) \cdot \psi^{n-3 k} \cdot n \cdot(n-1)} \times S_{0}(k, n)\right) \cdot V_{0}^{n}(y)\right] \\
& Q\left(V_{0}(y), p^{\prime}\right)=V_{0}(y)+\sum_{n=4}^{n=\infty}\left[\left(\frac{(-1)^{n} p^{\prime}}{V_{0}^{3}(0) \cdot \psi^{n-4} \cdot n \cdot(n-1)}+\sum_{k=2}^{m^{\prime}} \frac{(-1)^{n-k-1} p^{\prime k}}{V_{0}^{3 k}(0) \cdot \psi^{n-3 k-1} \cdot n \cdot(n-1)} \times S_{1}(k, n)\right) \cdot V_{0}^{n}(y)\right]
\end{aligned}
$$

Par ailleurs, la relation (5) conduit à écrire le potentiel $\varepsilon / 2\left(y, p^{\prime}\right)$ :

$$
\frac{\varepsilon}{2}\left(y, p^{\prime}\right)=a_{0} \cdot R\left(V_{0}(y), p^{\prime}\right)+a_{1} \cdot S\left(V_{0}(y), p^{\prime}\right)
$$


où l'on a posé :

$$
\begin{gathered}
R\left(V_{0}(y), p^{\prime}\right)=\frac{1}{p^{\prime}} \cdot B\left(V_{0}(y)\right) \cdot R^{\prime}\left(V_{0}(y), p^{\prime}\right) \\
S\left(V_{0}(y), p^{\prime}\right)=\frac{1}{p^{\prime}} \cdot B\left(V_{0}(y)\right) \cdot S^{\prime}\left(V_{0}(y), p^{\prime}\right) \\
R^{\prime}\left(V_{0}(y), p^{\prime}\right)=\sum_{n=3}^{n=\infty}\left[\left(\frac{(-1)^{n-1} p^{\prime}}{V_{0}^{3}(0) \cdot \psi^{n-3} \cdot(n-1)}+\sum_{k=2}^{m} \frac{(-1)^{n-k} p^{\prime k}}{V_{0}^{3 k}(0) \cdot \psi^{n-3 k} \cdot(n-1)} \times S_{0}(k, n)\right) \cdot V_{0}^{n-1}(y)\right] \\
S^{\prime}\left(V_{0}(y), p^{\prime}\right)=1+\sum_{n=4}^{n=\infty}\left[\left(\frac{(-1)^{n} p^{\prime}}{V_{0}^{3}(0) \cdot \psi^{n-4} \cdot(n-1)}+\sum_{k=2}^{m^{\prime}} \frac{(-1)^{n-k-1} p^{\prime k}}{V_{0}^{3 k}(0) \cdot \psi^{n-3 k-1} \cdot(n-1)} \times S_{1}(k, n)\right) \cdot V_{0}^{n-1}(y)\right]
\end{gathered}
$$

3.2 APPRONIMATIONS ASYMPTOTIQUES DES SOlUtions. - Pour obtenir les solutions asymptotiques, valables en hautes fréquences, du système $(4,5)$ on transforme celui-ci selon la méthode indiquée par Liouville et Schelkunoff $[32,33]$ par les changements de variable suivants :

$$
\begin{aligned}
K\left(V_{0}(y)\right) & =\sqrt{\frac{\psi \cdot V_{0}(y)}{\left[\psi+V_{0}(y)\right] \cdot V_{0}(0)^{3} \cdot p^{\prime}}} \\
u & =\int^{V_{0}(y)} p^{\prime} \cdot K\left(V_{0}(y)\right) \cdot \mathrm{d} V_{0}(y) \\
\hat{X}\left(V_{0}(y)\right) & =\frac{\varepsilon}{2}(y, p) \cdot\left[B\left(V_{0}(y)\right) \cdot \sqrt{\left.K\left(V_{0}(y)\right)\right]^{-1}}\right. \\
\hat{i} & =i \cdot \sqrt{K\left(V_{0}(y)\right)}
\end{aligned}
$$

afin d'obtenir le système homogène de type Sturm Liouville

$$
\begin{gathered}
\frac{\mathrm{d}^{2} \hat{X}}{\mathrm{~d} u^{2}}-\left[1+\frac{3 K_{(u)}^{\prime 2}}{4 K^{2}}-\frac{K_{\left(u^{2}\right)}^{\prime \prime}}{2 K}\right] \cdot \hat{X}=0 \\
\frac{\mathrm{d}^{2} \hat{i}}{\mathrm{~d} u^{2}}-\left[1-\frac{K_{(u)}^{\prime 2}}{4 K^{2}}+\frac{K_{(u)}^{\prime \prime}}{2 K}\right] \cdot \hat{i}=0 .
\end{gathered}
$$

Le système $(25,26)$ devient à coefficients constants et est soluble par l'approximation WKB [34] lorsque les termes entre crochets sont voisins de l'unité ; cette condition impose que soit respectée en tout point du canal l'inégalité :

$$
\left|\frac{I_{\mathrm{D}}}{p Z C_{\mathrm{ox}}}\left\{\frac{5}{16} \frac{\left[\frac{\mathrm{d}^{2} V_{0}(y)}{\mathrm{d} y^{2}}\right]^{2}}{\left[\frac{\mathrm{d} V_{0}(y)}{\mathrm{d} y}\right]^{3}}-\frac{1}{4} \frac{\left[\frac{\mathrm{d}^{3} V_{0}(y)}{\mathrm{d} y^{3}}\right]}{\left[\frac{\mathrm{d} V_{0}(y)}{\mathrm{d} y}\right]^{2}}\right\}\right| \ll 1 .
$$

Cette situation sera réalisée si la fréquence et les conditions de polarisation, sont telles que :

$$
|p| \gg \frac{7}{64} \frac{\psi}{\psi+V_{0}(0)} \frac{\mu_{0} \cdot V_{0}(0)}{L^{2}}\left[\frac{V_{0}(0)}{V_{0}(L)}\right]^{3}\left\{1-\left[\frac{V_{0}(L)}{V_{0}(0)}\right]^{2}\right\}
$$

On obtient, compte tenu des conditions au drain $(y=L)$ :

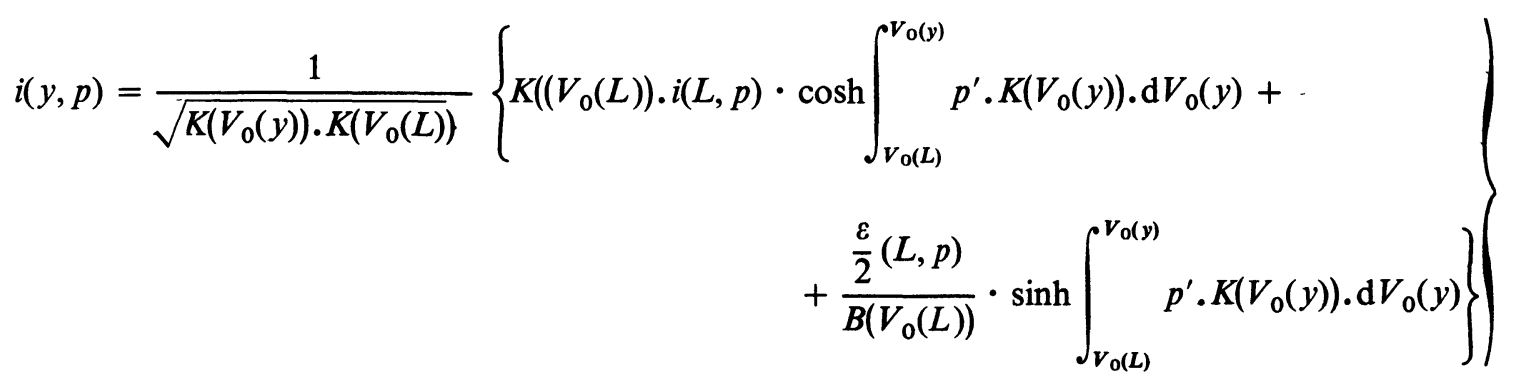




$$
\begin{aligned}
\frac{\varepsilon}{2}(y, p)=B\left(V_{0}(y)\right) \cdot \sqrt{\frac{K\left(V_{0}(y)\right)}{K\left(V_{0}(L)\right)}\left\{K\left(V_{0}(L)\right) \cdot i(L, p)\right.} \cdot \sinh \int_{V_{0}(L)}^{V_{0}(y)} p^{\prime} \cdot K\left(V_{0}(y)\right) \cdot \mathrm{d} V_{0}(y)+ \\
\left.\left.+\frac{\frac{\varepsilon}{2}(L, p)}{B\left(V_{0}(L)\right)} \cdot \cosh \int_{V_{0}(L)}^{V_{0}(y)} p^{\prime} \cdot K\left(V_{0}(y)\right) \cdot \mathrm{d} V_{0}(y)\right\} \cdot\right\}
\end{aligned}
$$

4. Expression des facteurs de la matrice admittance $y_{i j}$ " intrinsèque ». - 4.1 DéFINITION DE LA MATRICE " $y_{i j} \|$. - Le transistor MOS doit être considéré comme un dispositif à trois électrodes de commande (Fig. 2). Lorsqu'on applique sur la grille, le drain et la source des tensions $v_{\mathrm{g}}(p) / 2, v_{\mathrm{d}}(p) / 2, v_{\mathrm{r}}(p) / 2$, qui dans notre formalisme s'identifient à :

$$
\begin{aligned}
& \frac{v_{\mathrm{d}}(p)}{2}=-\frac{\varepsilon}{2}(L, p)+\frac{v_{\mathrm{g}}(p)}{2} \\
& \frac{v_{\mathrm{r}}(p)}{2}=-\frac{\varepsilon}{2}(0, p)+\frac{v_{\mathrm{g}}(p)}{2}
\end{aligned}
$$

et d'amplitudes suffisamment faibles pour pouvoir appliquer le principe de superposition, on définit une matrice admittance par l'égalité :

$$
\left[\begin{array}{c}
i_{\mathrm{g}}(p) \\
i(L, p) \\
-i(0, p)
\end{array}\right]=\left[\begin{array}{lll}
y_{11} & y_{12} & y_{13} \\
y_{21} & y_{22} & y_{23} \\
y_{31} & y_{32} & y_{33}
\end{array}\right]\left[\begin{array}{c}
v_{\mathrm{g}}(p) / 2 \\
-\frac{\varepsilon}{2}(L, p)+v_{\mathrm{g}}(p) / 2 \\
-\frac{\varepsilon}{2}(0, p)+v_{\mathrm{g}}(p) / 2
\end{array}\right] .
$$

Le régime de fonctionnement est complètement déterminé en associant à cette relation matricielle la loi de conservation du courant qui s'exprime par :

$$
i(0, p)-i(L, p)-i_{\mathrm{g}}(p)=0
$$

puisqu'on néglige le courant de substrat d'après l'hypothèse du faible dopage de ce dernier.

La méthode de détermination des facteurs $y_{i j}$ est basée sur l'utilisation de la relation matricielle (33) et des expressions de la tension $\varepsilon / 2(y, p)$ et du courant $i(y, p)$. Chaque admittance est calculée en tenant compte des conditions aux limites sur les électrodes que nous introduisons sous forme de l'identité :

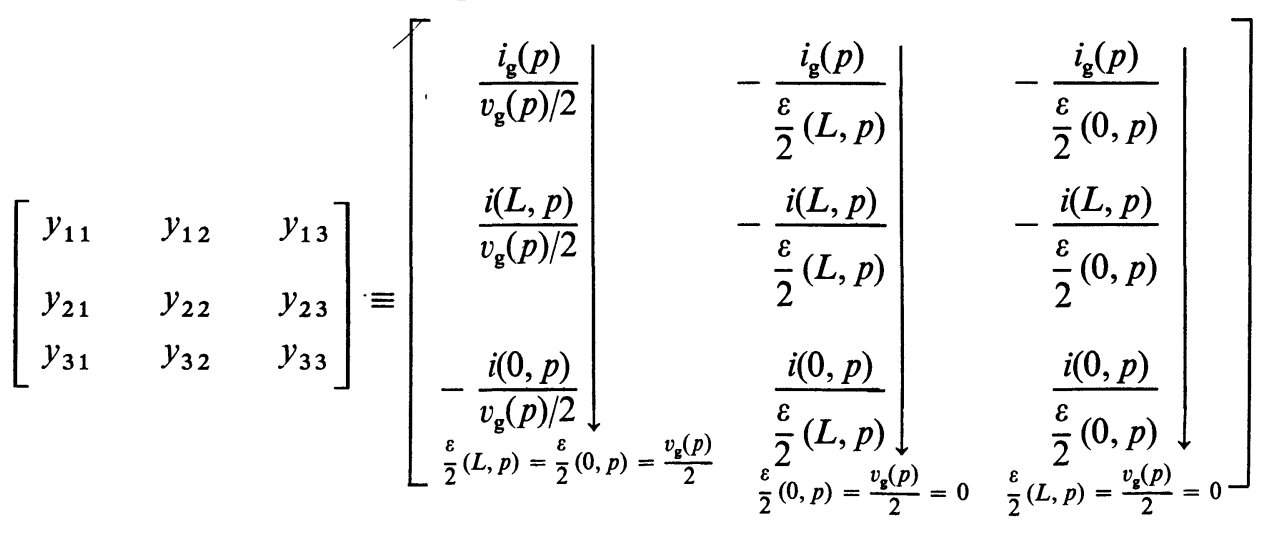

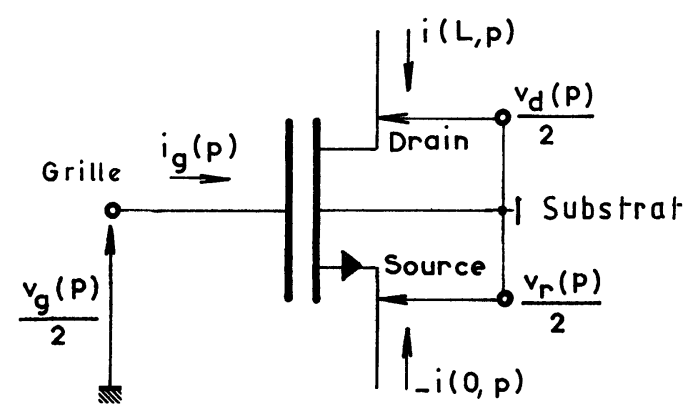

Fig. 2. - Symboles de définition de la matrice admittance.

[Symbols defining the admittance matrix.] 
4.1.1 Expression des facteurs $y_{i j}$ au moyen de séries. - Chaque colonne de (35) conduit à une relation de liaison entre $a_{0}$ et $a_{1}$.

$$
\begin{aligned}
& \text { 1re colonne }: a_{1}=a_{0} \cdot \frac{R\left(V_{0}(0), p^{\prime}\right)-R\left(V_{0}(L), p^{\prime}\right)}{S\left(V_{0}(L), p^{\prime}\right)-S\left(V_{0}(0), p^{\prime}\right)} \\
& \text { 2e colonne }: a_{1}=a_{0} \cdot \frac{R\left(V_{0}(0), p^{\prime}\right)}{S\left(V_{0}(0), p^{\prime}\right)} \\
& \text { 3e colonne }: a_{1}=a_{0} \cdot \frac{R\left(V_{0}(L), p^{\prime}\right)}{S\left(V_{0}(L), p^{\prime}\right)}
\end{aligned}
$$

Compte tenu des expressions $i(L, p), i(0, p), \varepsilon / 2(L, p), \varepsilon / 2(0, p)$ déduites de (13) et (16), des conditions de liaison (36) à (38), et de la possibilité de normalisation par introduction des variables suivantes :

$$
\begin{aligned}
\hat{y}_{i j} & =\frac{y_{i j}}{\mu_{0} \frac{Z}{L} C_{\mathrm{ox}} \cdot V_{0}(0)} \\
\eta & =\frac{V_{0}(L)}{V_{0}(0)} \\
\pi & =\frac{V_{0}(L)}{\psi} \\
\lambda & =\frac{V_{0}(0)}{L E_{0}}
\end{aligned}
$$

et des quantités :

$$
\begin{aligned}
& \hat{P}_{0}\left(\eta, \pi, p^{\prime}\right)=1+\sum_{n=3}^{\infty}\left[\frac{(-1)^{n-1} p^{\prime}}{n(n-1)} \frac{\pi^{n-3}}{\eta^{n-3}}+\sum_{k=2}^{m} \frac{(-1)^{n-k} p^{\prime k}}{n(n-1)} S_{0}(k, n) \frac{\pi^{n-3 k}}{\eta^{n-3 k}}\right] \\
& \hat{P}_{L}\left(\eta, \pi, p^{\prime}\right)=1+\sum_{n=3}^{\infty}\left[\frac{(-1)^{n-1} p^{\prime}}{n(n-1)} \cdot \eta^{n} \cdot \pi^{n-3}+\sum_{k=2}^{m} \frac{(-1)^{n-k} p^{\prime k}}{n(n-1)} S_{0}(k, n) \eta^{n} \pi^{n-3 k}\right] \\
& \hat{Q}_{0}\left(\eta, \pi, p^{\prime}\right)=1+\sum_{n=4}^{\infty}\left[\frac{(-1)^{n} p^{\prime}}{n(n-1)} \frac{\pi^{n-4}}{\eta^{n-4}}+\sum_{k=2}^{m^{\prime}} \frac{(-1)^{n-k-1} p^{\prime k}}{n(n-1)} S_{1}(k, n) \frac{\pi^{n-3 k-1}}{\eta^{n-3 k-1}}\right] \\
& \hat{Q}_{L}\left(\eta, \pi, p^{\prime}\right)=1+\sum_{n=4}^{\infty}\left[\frac{(-1)^{n} p^{\prime}}{n(n-1)} \eta^{n-1} \pi^{n-4}+\sum_{k=2}^{m^{\prime}} \frac{(-1)^{n-k-1} p^{\prime k}}{n(n-1)} S_{1}(k, n) \cdot \eta^{n-1} \cdot \pi^{n-3 k-1}\right] \\
& \hat{R}_{L}^{\prime}\left(\eta, \pi, p^{\prime}\right)=\sum_{n=3}^{\infty}\left[\frac{(-1)^{n-1} p^{\prime}}{n-1} \eta^{n} \pi^{n-3}+\sum_{k=2}^{m} \frac{(-1)^{n-k} p^{\prime k}}{n-1} S_{0}(k, n) \eta^{n} \pi^{n-3 k}\right] \\
& \hat{R}_{0}^{\prime}\left(\eta, \pi, p^{\prime}\right)=\sum_{n=3}^{\infty}\left[\frac{(-1)^{n-1} p^{\prime}}{n-1} \frac{\pi^{n-3}}{\eta^{n-4}}+\sum_{k=2}^{m} \frac{(-1)^{n-k} p^{\prime k}}{n-1} S_{0}(k, n) \frac{\pi^{n-3 k}}{\eta^{n-3 k-1}}\right] \\
& \hat{S}_{0}^{\prime}\left(\eta, \pi, p^{\prime}\right)=1+\sum_{n=4}^{\infty}\left[\frac{(-1)^{n} p^{\prime}}{n-1} \frac{\pi^{n-4}}{\eta^{n-4}}+\sum_{k=2}^{m^{\prime}} \frac{(-1)^{n-k-1} p^{\prime k}}{n-1} S_{1}(k, n) \frac{\pi^{n-3 k-1}}{\eta^{n-3 k-1}}\right] \\
& \hat{S}_{L}^{\prime}\left(\eta, \pi, p^{\prime}\right)=1+\sum_{n=4}^{\infty}\left[\frac{(-1)^{n} p^{\prime}}{n-1} \eta^{n-1} \pi^{n-4}+\sum_{k=2}^{m^{\prime}} \frac{(-1)^{n-k-1} p^{\prime k}}{n-1} S_{1}(k, n) \cdot \eta^{n-1} \cdot \pi^{n-3 k-1}\right] \\
& \hat{B}_{0}(\eta, \pi, \lambda)=\frac{(\pi / \eta)^{2}}{A(\eta, \pi, \lambda)}\left[\frac{1}{1+\eta / \pi}-\lambda . A(\eta, \pi, \lambda)\right]^{-1} \\
& \hat{B}_{L}(\eta, \pi, \lambda)=\frac{(\pi / \eta)^{2}}{A(\eta, \pi, \lambda)}\left[\frac{1}{1+1 / \pi}-\lambda . A(\eta, \pi, \lambda)\right]^{-1}
\end{aligned}
$$

avec

$$
A(\eta, \pi, \lambda)=\left[1-\eta+\frac{\eta}{\pi} \log \frac{1+\pi}{1+\pi / \eta}\right][(1-\eta) \lambda+1]^{-1} .
$$

Les expressions des paramètres $\hat{y}_{i j}$ sont :

$$
\hat{y}_{22}\left(p^{\prime}\right)=\frac{\eta p^{\prime}\left[\hat{Q}_{0}\left(\eta, \pi, p^{\prime}\right) \cdot \hat{R}_{0}^{\prime}\left(\eta, \pi, p^{\prime}\right)-\hat{P}_{0}\left(\eta, \pi, p^{\prime}\right) \cdot \hat{S}_{0}^{\prime}\left(\eta, \pi, p^{\prime}\right)\right]}{\hat{B}_{L}(\eta, \pi, \lambda)\left[\hat{R}_{L}^{\prime}\left(\eta, \pi, p^{\prime}\right) \cdot \hat{S}_{0}^{\prime}\left(\eta, \pi, p^{\prime}\right)-\hat{S}_{L}^{\prime}\left(\eta, \pi, p^{\prime}\right) \cdot \hat{R}_{0}^{\prime}\left(\eta, \pi, p^{\prime}\right)\right]}
$$




$$
\begin{aligned}
\hat{y}_{23}\left(p^{\prime}\right)=\frac{p^{\prime}\left[\eta \cdot \hat{S}_{L}^{\prime}\left(\eta, \pi, p^{\prime}\right) \cdot \hat{P}_{L}\left(\eta, \pi, p^{\prime}\right)-\hat{Q}_{L}\left(\eta, \pi, p^{\prime}\right) \cdot \hat{R}_{L}^{\prime}\left(\eta, \pi, p^{\prime}\right)\right]}{\hat{B}_{0}(\eta, \pi, \lambda)\left[\hat{R}_{L}^{\prime}\left(\eta, \pi, p^{\prime}\right) \cdot \hat{S}_{0}^{\prime}\left(\eta, \pi, p^{\prime}\right)-\hat{S}_{L}^{\prime}\left(\eta, \pi, p^{\prime}\right) \cdot \hat{R}_{0}^{\prime}\left(\eta, \pi, p^{\prime}\right)\right]} \\
\hat{y}_{32}\left(p^{\prime}\right)=\frac{p^{\prime}\left[\eta \cdot \hat{P}_{0}\left(\eta, \pi, p^{\prime}\right) \cdot \hat{S}_{0}^{\prime}\left(\eta, \pi, p^{\prime}\right)-\hat{Q}_{0}\left(\eta, \pi, p^{\prime}\right) \cdot \hat{R}_{0}^{\prime}\left(\eta, \pi, p^{\prime}\right)\right]}{\hat{B}_{L}(\eta, \pi, \lambda)\left[\hat{R}_{L}^{\prime}\left(\eta, \pi, p^{\prime}\right) \cdot \hat{S}_{0}^{\prime}\left(\eta, \pi, p^{\prime}\right)-\hat{S}_{L}^{\prime}\left(\eta, \pi, p^{\prime}\right) \cdot \hat{R}_{0}^{\prime}\left(\eta, \pi, p^{\prime}\right)\right]} \\
\hat{y}_{33}\left(p^{\prime}\right)=\frac{\eta \cdot p^{\prime}\left[\hat{R}_{L}^{\prime}\left(\eta, \pi, p^{\prime}\right) \cdot \hat{Q}_{0}\left(\eta, \pi, p^{\prime}\right)-\hat{P}_{0}\left(\eta, \pi, p^{\prime}\right) \cdot \hat{S}_{L}^{\prime}\left(\eta, \pi, p^{\prime}\right)\right]}{B_{0}(\eta, \pi, \lambda)\left[\hat{R}_{L}^{\prime}\left(\eta, \pi, p^{\prime}\right) \cdot \hat{S}_{0}^{\prime}\left(\eta, \pi, p^{\prime}\right)-\hat{S}_{L}^{\prime}\left(\eta, \pi, p^{\prime}\right) \cdot \hat{R}_{0}^{\prime}\left(\eta, \pi, p^{\prime}\right)\right]} \\
\hat{y}_{11}=-\hat{y}_{21}+\hat{y}_{32}+\hat{y}_{33} \\
\hat{y}_{21}=-\hat{y}_{22}-\hat{y}_{23} \\
\hat{y}_{13}=-\hat{y}_{23}-\hat{y}_{33} \\
\hat{y}_{12}=-\hat{y}_{22}-\hat{y}_{32} \\
\hat{y}_{31}=-\hat{y}_{33}-\hat{y}_{32} .
\end{aligned}
$$

4.2 EXPRESSIONS ASYMPTOTIQUES DES FACTEURS $y_{i j}$. - La méthode de détermination des expressions asymptotiques, dans la gamme des fréquences satisfaisant l'inégalité (28), des facteurs de la matrice $y_{i j}$ consiste à utiliser d'une part l'identité matricielle (35) et les conditions aux limites au drain et à la source, d'autre part les expressions (29) et (30) du courant et de la tension.

On obtient sous forme normalisée les paramètres du montage à source commune :

$$
\begin{aligned}
& \hat{y}_{22}\left(p^{\prime}\right)=-E\left(\eta, \pi, \lambda, p^{\prime}\right) \cdot \frac{1}{\tanh \left[\mathcal{F}\left(p^{\prime}, \eta, \lambda, \pi\right)\right]} \\
& \hat{y}_{21}\left(p^{\prime}\right)=-\left[\hat{y}_{22}\left(p^{\prime}\right)+\frac{H\left(\eta, \pi, \lambda, p^{\prime}\right)}{\sinh \left[\mathscr{F}\left(\eta, \pi, \lambda, p^{\prime}\right)\right]}\right] \\
& \hat{y}_{12}\left(p^{\prime}\right)=\hat{y}_{22}\left(p^{\prime}\right)\left\{D\left(\eta, \pi, \lambda, p^{\prime}\right) \cdot \cosh \left[\mathcal{F}\left(\eta, \pi, \lambda, p^{\prime}\right)\right]-1\right\}+C\left(\eta, \pi, \lambda, p^{\prime}\right) \cdot \sinh \left[\mathcal{F}\left(\eta, \pi, \lambda, p^{\prime}\right)\right] \\
& \hat{y}_{11}\left(p^{\prime}\right)=\hat{y}_{21}\left(p^{\prime}\right)\left\{D\left(\eta, \pi, \lambda, p^{\prime}\right) \cdot \cosh \left[\mathcal{F}\left(\eta, \pi, \lambda, p^{\prime}\right)\right]-1\right\}-C\left(\eta, \pi, \lambda, p^{\prime}\right) \cdot \sinh \left[\mathcal{F}\left(\eta, \pi, \lambda, p^{\prime}\right)\right]
\end{aligned}
$$

avec :

$$
\begin{aligned}
& E\left(\eta, \pi, \lambda, p^{\prime}\right)=A(\eta, \pi, \lambda) \cdot\left(\frac{\eta}{\pi}\right)^{3 / 2} \sqrt{p^{\prime}\left(1+\frac{1}{\pi}\right)}\left[\frac{1}{1+1 / \pi}-\lambda \cdot A(\eta, \pi, \lambda)\right] \\
& D\left(\eta, \pi, \lambda, p^{\prime}\right)=\left(\frac{\eta+\pi}{1+\pi}\right)^{1 / 4} \\
& H\left(\eta, \pi, \lambda, p^{\prime}\right)=E\left(\eta, \pi, \lambda, p^{\prime}\right) \cdot D\left(\eta, \pi, \lambda, p^{\prime}\right)\left[\frac{\left.\frac{1}{1+\eta / \pi}-\lambda \cdot A(\eta, \pi, \lambda)\right]}{\frac{1}{1+1 / \pi}-\lambda \cdot A(\eta, \pi, \lambda)}\right] \\
& C\left(\eta, \pi, \lambda, p^{\prime}\right)=E\left(\eta, \pi, \lambda, p^{\prime}\right) \cdot D\left(\eta, \pi, \lambda, p^{\prime}\right) \\
& \mathcal{F}\left(\eta, \pi, \lambda, p^{\prime}\right)=\sqrt{p^{\prime} \cdot \frac{\eta}{\pi}}\left[\eta \sqrt{1+\frac{1}{\pi}}-\sqrt{1+\frac{\eta}{\pi}}-\frac{\eta}{\pi} \log \left(\frac{1+\sqrt{1+\frac{1}{\pi}}}{\sqrt{\frac{1}{\eta}}+\sqrt{\frac{1}{\eta}+\frac{1}{\pi}}}\right)\right] .
\end{aligned}
$$

5. Variations des paramètres normalisés. - Les facteurs normalisés $y_{i j}$ ont été calculés premièrement par application des relations (63) à (66). La plage d'utilisation de ces relations, déduite de (27), a été limitée au cas où l'erreur sur les modules des $y_{i j}$ est inférieure à $5 \%$, condition qui se traduit par une limitation, en valeur minimale d'une pulsation $\hat{\omega}$ en fonction des paramètres $\eta, \lambda, \pi$. Cette pulsation réduite $\hat{\omega}$ est définie par la relation :

$$
\hat{\omega}=\frac{p^{\prime}}{j} A^{2}(\eta, \lambda, \pi) \cdot \frac{\eta^{2}}{\pi^{2}} .
$$

Elle s'identifie à la pulsation normalisée utilisée de façon classique dans la littérature et qui a été introduite pour la première fois par Geurst et Nunnink [24] :

$$
\hat{\omega}=\omega \frac{L^{2}}{\mu_{0}\left(V_{\mathrm{G}}^{\prime}-V_{\mathrm{R}}\right)} \text {. }
$$


Physiquement, dans le cadre de l'hypothèse de mobilité constante ( $\psi$ et $L E_{0}$ tendant vers l'infini) $\hat{\omega}$ représente le rapport entre la fréquence du signal d'excitation et la fréquence de coupure du gain en courant en hautes fréquences cette dernière étant égale au rapport de la transconductance

$$
\left[\mu_{0} \frac{Z}{L} C_{\mathrm{ox}}\left(V_{\mathrm{G}}^{\prime}-V_{\mathrm{R}}\right)\right]
$$

à $2 \pi$ fois la capacité de l'oxyde de grille $\left(2 \pi . Z L C_{\text {ox }}\right)$. La figure 3 représente la limite fréquentielle d'application des relations (63) à (68). Par ailleurs dans le domaine fréquentiel complémentaire du précédent, les paramètres $\hat{y}_{i j}$ sont calculés par détermination, au moyen de récurrences, des divers termes des séries intervenant dans les expressions (54) à (62). Les évolutions des facteurs $\hat{y}_{i j}$, qui ne dépendent que des quantités $\eta, \pi, \lambda, \hat{\omega}$ dont les définitions sont rappelées dans le tableau I, sont représentées dans le plan complexe (Figs. 4 et 5).

Les diverses courbes - relatives à chaque valeur de $\eta$ - sont paramétrées par la pulsation réduite $\hat{\omega}$. D'autre part, pour traduire globalement les effets de réductions longitudinale et transversale de mobilité, les graphiques ont été tracés en conservant les quantités $\pi / \eta$ et $\lambda . \eta$, c'est-à-dire les rapports $V_{G}^{\prime}-V_{R} / \psi$ et

Tableau I. - Définition des paramètres.

[(Parameters definition).]

Tensions continues $\left\{\begin{array}{l}\text { Grille substrat (effective) } \\ \text { Source substrat } \\ \text { Drain substrat }\end{array}\right.$

Potentiel $V_{0}(y)\left\{\begin{array}{l}\text { Côté source } \\ \text { Côté drain }\end{array}\right.$

Variables de normalisation

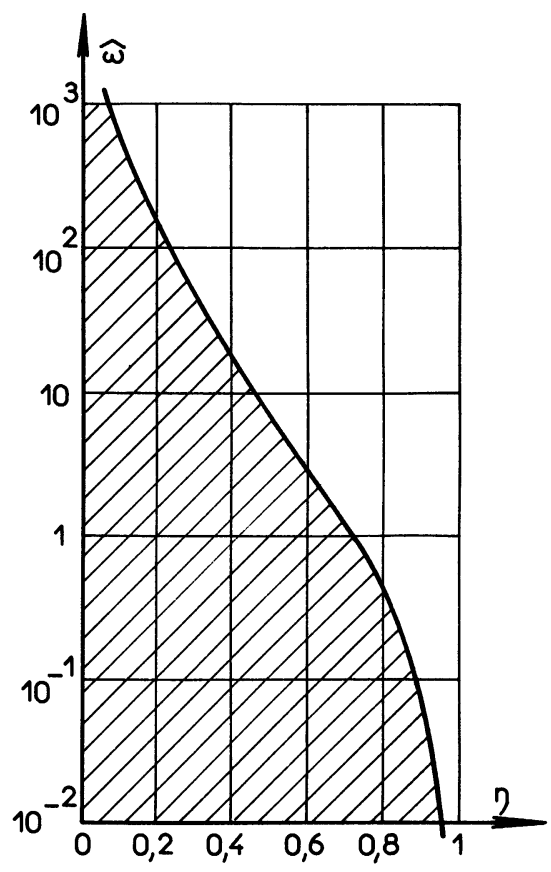

Fig. 3. - Domaine de validité des relations (63) à (66) (erreur sur $\left|\hat{y}_{i j}\right|$ inférieure à $5 \%$ ). Cas particuliers ou $\lambda=0$ et $\pi=0$.

[Range of validity of expression (63) to (66). Error on $\left|\hat{y}_{i j}\right|<5 \%$. Particular case where : $\lambda=0 ; \pi=0$.]

Régime non saturé

Régime saturé

$$
\begin{aligned}
& \text { - } V_{\mathrm{G}}^{\prime} \\
& V_{\mathrm{D}} \quad V_{\mathrm{p}} \\
& V_{0}(0)=V_{G}^{\prime}-V_{R} \\
& V_{0}(L)=V_{\mathrm{G}}^{\prime}-V_{\mathrm{D}} \quad V_{0}(L)=V_{\mathrm{G}}^{\prime}-V_{\mathrm{p}} \\
& \eta=\frac{V_{\mathrm{G}}^{\prime}-V_{\mathrm{D}}}{V_{\mathrm{G}}^{\prime}-V_{\mathrm{R}}} \quad \eta=\frac{V_{\mathrm{G}}^{\prime}-V_{\mathrm{p}}}{V_{\mathrm{G}}^{\prime}-V_{\mathrm{R}}} \\
& \pi=\frac{V_{\mathrm{G}}^{\prime}-V_{\mathrm{D}}}{\psi} \quad \pi=\frac{V_{\mathrm{G}}^{\prime}-V_{\mathrm{p}}}{\psi} \\
& \lambda=\frac{V_{\mathrm{G}}^{\prime}-V_{\mathbf{R}}}{L E_{0}} \\
& \hat{\omega}=\frac{\omega L^{2}}{\mu_{0}\left(V_{G}^{\prime}-V_{R}\right)} \\
& \hat{y}_{i j}=y_{i j} \frac{1}{\mu_{0} \frac{Z}{L} C_{\mathrm{ox}}\left(V_{\mathrm{G}}^{\prime}-V_{\mathrm{R}}\right)}
\end{aligned}
$$



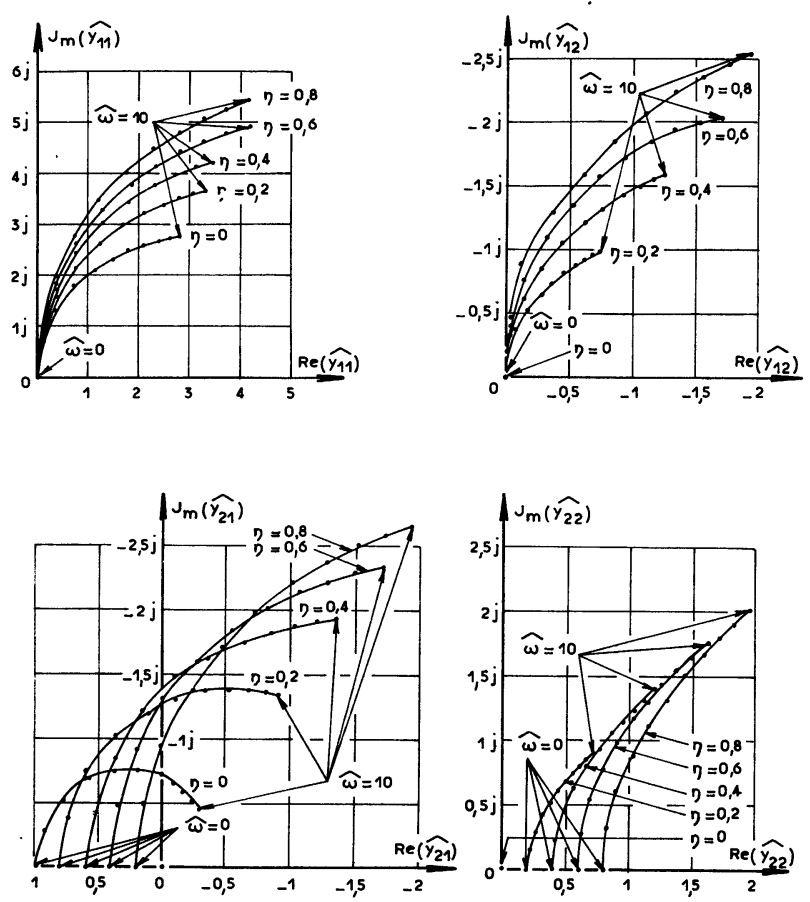

Fig. 4. - Variations des paramètres normalisés $\hat{y}_{i j}: \pi=0 ; \lambda=0$. $\hat{\omega}$ varie de 0 à 10 avec un pas unitaire (la caractéristique de $\eta=0$ correspond au régime de saturation).

[Variations in normalised parameters $\hat{y}_{i j}: \pi=0 ; \lambda=0 . \hat{\omega}$ varies from 0 . to 10 . in steps of 1 (saturation range for $\eta=0$ ).]

$\left(V_{\mathrm{G}}^{\prime}-V_{\mathrm{D}}\right) / L E_{0}\left(\right.$ ou $\left.\left(V_{\mathrm{G}}^{\prime}-V_{\mathrm{p}}\right) / L E_{0}\right)$ constants. Enfin les réseaux relatifs au fonctionnement en régime pincé du transistor ont été calculés dans le cadre de l'hypothèse de saturation parfaite, conduisant à la valeur du paramètre $\eta$ - et par suite du potentiel $V_{\mathrm{p}}-$ qui vérifie l'équation implicite :

$$
A(\eta, \pi, \lambda)=\frac{\pi}{\lambda(\pi+1)} .
$$

Cette hypothèse revient à négliger les effets de modulation de la longueur effective du canal en régime saturé [35].

Afin de mettre en évidence les effets de réduction de mobilité, nous avons tout d'abord reporté sur la figure 4 les facteurs $\hat{y}_{i j}$ dans le cas de mobilité constante; les résultats obtenus sont identiques à ceux proposés antérieurement par d'autres auteurs [24]. D'autre part, lorsque la mobilité est réduite sous l'action des coefficients $\lambda$ et $\pi$ (Fig. 5), on constate que l'amplitude des parties réelles et imaginaires est modifiée. En particulier dans l'exemple traité, qui correspond à un transistor MOS dans lequel la tension effective $V_{0}(0)$ reste inférieure au quart du produit de la longueur $L$ par le champ critique $E_{0}$, l'admittance de transfert directe $y_{21}$ est la plus affectée, surtout si le transistor est polarisé en régime de pincement. Cet effet, qui entraîne une dégradation
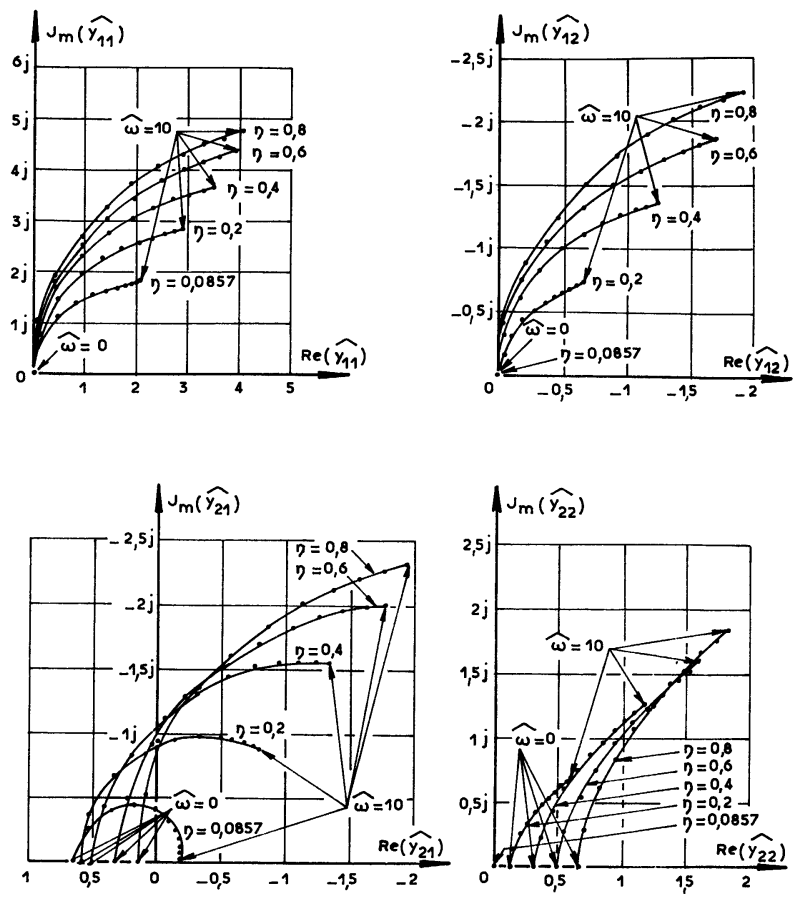

Fig. 5. - Variations des paramètres normalisés $\hat{y}_{i j}: \pi=0,2$; $\lambda=0,02 / \eta$. $\hat{\omega}$ varie de 0 à 10 avec un pas unitaire (la caractéristique $=0,0857$ correspond au régime de saturation).

[Variations of normalised parameters $\hat{y}_{i j}: \pi=0.2 \eta ; \lambda=0.02 / \eta$. $\hat{\omega}$ varies from 0 . to 10 . in steps of 1 (saturation range for $\eta=0.0857)$.]

notable du gain en puissance, montre que les théories classiques ne sont pas adaptées à l'analyse du comportement des transistors MOS à canal court dans lesquels les porteurs peuvent atteindre la vitesse limite dans une fraction du canal.

6. Conclusion. - L'analyse théorique qui vient d'être développée a permis d'exprimer les facteurs de la matrice admittance intrinsèque $\left[y_{i j}\right]$ du transistor MOS lorsque les effets de la réduction de mobilité sous l'action des deux composantes du champ électrique sont pris en compte. Rappelons que ces mécanismes de modification de vitesse des porteurs, bien analysés en ce qui concerne le régime statique $d u$ transistor MOS, n'avaient jamais été pris en considération dans l'évaluation des performances en hautes fréquences. L'originalité du formalisme utilisé a permis de présenter les résultats sous forme normalisée par introduction de quatre variables réduites $\eta, \lambda$, $\pi, \hat{\omega}$ qui dépendent à la fois des conditions de polarisation - de grille $V_{\mathrm{G}}^{\prime}$, de source $V_{\mathrm{R}}$ et de drain $V_{\mathrm{D}}$ des dimensions géométriques - largeur $Z$, longueur $L$, épaisseur d'oxyde $x_{0}$ - des caractéristiques physiques - mobilité à champ nul $\mu_{0}$, champs critiques longitudinal $E_{0}$ et transversal $E_{\mathrm{c}}$, charges parasites $Q_{\mathrm{ss}}$ et différence des travaux de sortie $\varphi_{\mathrm{MS}}$ - et de la pulsation des signaux d'excitation $\omega$. 
Il serait possible de simplifier les expressions proposées en les limitant au premier ou au deuxième ordre pour conduire à un schéma équivalent simplifié dont le domaine de validité fréquentiel resterait à déterminer. Par ailleurs, pour rendre compte de façon précise du comportement en hautes fréquences des structures réelles, il convient de rajouter à la théorie développée dans cet article deux effets : le premier est relatif à l'influence des éléments parasites, le deuxième est lié à la réduction de la longueur apparente du canal en régime de pincement [35].
Ce modèle théorique qui a l'avantage de prendre en compte l'effet de la tension source substrat a déjà été appliqué à l'établissement des modèles [36] ou à la conception [9-12] de transistors MOS double grille à canal court. Dans un futur article, nous l'utiliserons d'une part pour décrire les particularités de fonctionnement en hautes fréquences du transistor MOS à canal vertical et d'autre part pour concevoir des amplificateurs à large bande utilisant ce dispositif.

\section{Bibliographie}

[1] BuRnS, J. R., High frequency characteristics of the Insulated - Gate Field Effect Transistor. R.C.A. Rev. (1967) 385-418.

[2] Wallmark, J. T., Johnson, H., Field Effect Transistor - II High Frequency Circuits (Prentice Hall In.) 1966.

[3] Carlson, F. M., Application consideration for the VHF MOS FET - RCA Note AN 3193 (1966)

[4] Dawson, R. H., Preisig, J. O., Stevenson, D. M., UHF MOS integrated circuits, Research and Development technical report ECOM O 252 F $R C A$ Solid State Division (1971)

[5] SigG, H. J., Vendelin, G. D., Cauge, T. P., Kocsis, J., DMOS Transistor for microwave applications. IEEE transElectron. Devices 19 (1972) 45, 53.

[6] Beneking, H., Kasugai, H., Gigahertz P channel enhancement silicon MOSFET with non over lapping gate. IEEE J. Solid State Circuits 5 (1970).

[7] Stephenson, M. D., Wide Bandwidth Integrated circuit Amplifiers Using FET'S Ph. D. Thesis - University of Southampton 1973.

[8] Yu, S. Y., Ou. YANG, P., New V. Groove Double Diffused MOS (V. MOS), Electron. Lett. 12 (1976) 605.

[9] Zamorano, M., Contribution à l'étude des transistors MOS : Structures à double grille et structures de puissance à canal vertical. Thèse de $3^{\mathrm{e}}$ Cycle, Université Paul-Sabatier, Toulouse, juillet 1977.

[10] Thouy, M., Rossel, P., Caractérisation H. F. du Micro MOS. Note technique LETI-CEA CENG MEA No 1270 janvier 1978.

[11] Ronen, R. S., Strauss, L., The Silicon on Sapphire Tetrode, Some, Small Signal Feature L.F. to U.H.F., IEEE Trans. Electron Devices 21 (1974).

[12] Rossel, P., Suat, J. P., Martinot, H., Morin, G., Zamorano, M., Conception et réalisation de tétrodes MOS sur isolant destinées à l'amplification en hautes fréquences. Contrat DGRST no 75-7 0690 novembre 1976.

[13] Heng, T. M. S., OAKes, J. G., Tremere, D. A., Vertical Channel Metal-Oxyde Silicon Field Effect Transistor Contract No N 00014 - 74 C - 012, Off. Nav. Res. (1974).

[14] OAkes, J. G., Wickstrom, R. A., Tremere, D. A., Heng, T. M. S., A power silicon microwave MOS transistor IEEE Trans. Microwave Theory Tech. 24 (1976) 305, 311.

[15] OxNer, E. D., A New Technology Takes ON HF Power Bipolars, Microwave System News (1976) 107, 110.

[16] VAN DER ZIEL, A., ERO, J. W., Small signal high frequency theory of field effect transistors. IEEE Trans. Electron Devices (1964) 128-135.

[17] BURNs, J. R., High frequency characteristics of the insulated gate field effect transistor. R.C.A. Rev. (1967) 385-418.

[18] VAN NiELEN, J. A., A simple and accurate approximation to the high frequency characteristics of insulated gate field effect transistor. Solid State Electron., 12 (1969) 826-829.
[19] Rao, P. S., A theoretical study of excess noise in MOS FET's $\mathrm{Ph}$. D. Thesis, University of Minnesota, June 1970.

[20] Hopkins, R. S. H., High frequency (y) parameters of the MOS transistor $\mathrm{Ph}$. D. Thesis, Rutgers University, June 1970.

[21] Cherry, E. M., Small signal high frequency response of the insulated gate field effect transistor. IEEE Trans. Electron Devices, ED-17 (1970) 569-577.

[22] Haslett, J. W., Trofimenkoff, E. N., Small signal high frequency equivalent circuit for the MOS field effect transistor. Proc. IEEE 116 (1969) 699-702.

[23] Geurst, J. A., Calculation of high frequency characteristics of thin film transistor. Solid State Electron. 8 (1965) 88-90.

[24] Geurst, J. A., Nunnink, A. H. J. C., Numerical data on the high frequency characteristics of thin film transistors. Solid State Electron. 8 (1965) 769-771.

[25] Das, M. B., Generalized high frequency network theory of field effect transistors. Proc. IEEE 114 (1967).

[26] DAS, M. B., High frequency network properties of MOS transistor including the substrate resistivity effects. IEEE Trans. Electron Devices (1969) ED-12 1049-1069.

[27] Rossel, P., Propriétés statiques et dynamiques du transistor à effet de champ à grille isolée. Thèse de Doctorat ès Sciences, $n^{\circ}$ 529, 1973, Université Paul-Sabatier, Toulouse.

[28] Martinot, H., Rossel, P., High frequency properties of MOS transistor. Third European Solid State Device Research Conference, ESSDERC 1973, Munich, Germany.

[29] Martinot, H., Rossel, P., VassiliefF, G., Mobility parameters and MOS transistors properties. Electron Lett. 8 (1972) 599-600

[30] Pronontarios, E. N., Wing, O., Theory of non uniform RC lines, IEEE Trans. Commun. Technol. 14 (1967) 2-12.

[31] Pronontarios, E. N., Wing, O., Computation of the step response of a general non uniform $\mathrm{RC}$ distributed network. IEEE Trans. Commun. Technol. 144 (1967) 219-221.

[32] Shelkunoff, S. A., Applied mathematics for engineers and scientists (D. Van Nostrand), 1965, ch. XX, pp. 389-416.

[33] Rossel, P., Méthode de calcul des paramètres HF des transistors MOS en régime de non-pincement. Electron. Lett. 8 (1972) 614-617.

[34] Cunningham, W. J., Introduction to non linear analysis (Mc. Graw Hill Book Company Inc.) 1958, ch. IX.

[35] Rossel, P., Martinot, H., Modèle phénoménologique du transistor MOS en régime de saturation. Electron. Lett. 9 (1973).

[36] Belmas, M., Propriétés statiques en hautes fréquences des transistors MOS à double grille. Thèse de $3^{\mathrm{e}}$ Cycle, Université Paul-Sabatier, Toulouse no 1624,8 juillet 1974. 